\title{
EFFECT OF HEAT TREATMENT ON THE MECHANICAL
}

\section{PROPERTIES AND FRACTURE BEHAVIOR OF TUNGSTEN HEAVY}

\author{
ALLOYS

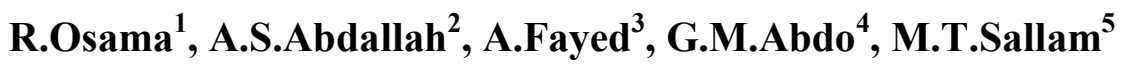 \\ ${ }^{I}$ Eng Researcher, Technical Research Center, Cairo, Egypt \\ ${ }^{2}$ Dr.Eng Senior Researcher, Technical Research Center, Cairo, Egypt \\ ${ }^{3}$ Dr.Eng, Material Science and Technology department, Military Technical College, Cairo, Egypt \\ ${ }^{4}$ Dr.Eng. head of Technical Researcher Center, Technical Research Center, Cairo, Egypt \\ ${ }^{5}$ Prof. Head of Material Science and Technology department, Military Technical college, Cairo, Egypt
}

\begin{abstract}
The objective of this experimental study is to investigate the effect of heat treatment on the mechanical properties and the fracture behavior of the liquid phase sintered (LPS) tungsten heavy alloys. For this purpose, tungsten alloy with three different compositions $93 \% \mathrm{~W}-4.9 \% \mathrm{Ni}-2.1 \% \mathrm{Fe}, \quad 91 \% \mathrm{~W}-6 \% \mathrm{Ni}-3 \% \mathrm{Co}$ and $90.5 \% \mathrm{~W}-5.6 \% \mathrm{Ni}-1.4 \% \mathrm{Fe}-1.5 \% \mathrm{Co}$ were prepared by conventional powder metallurgy route. Elemental powders were mixed using planetary mixer for 5 hours to ensure suitable homogeneity. Uni-axial compaction pressure of $200 \mathrm{MPa}$ was applied to obtain standard tensile and impact specimens. Vacuum liquid phase sintering was carried out at $1500^{\circ} \mathrm{C}$ for 60 minutes. The sintered specimens were then heat treated, at $1100^{\circ} \mathrm{C}$ for 60 min, in an atmosphere of dynamic argon gas, and then water quenched. The effect of this heat treatment was characterized in terms of hardness, impact resistance and tensile properties and compared to those properties for samples in the as sintered state. The application of the prescribed heat treatment on these heavy alloys with the adopted chemical compositions provided a remarkable improvements in the mechanical properties. The tensile fracture behavior of these alloys with was studied. By scanning electron microscope, the obtained micrographs of the fractured surfaces showed that, by the application of the prescribed heat treatment, the fracture modes changed from brittle intergranular separations to matrix failure and tungsten cleavage which corresponds to a net improvement in mechanical properties, especially in the alloys where cobalt is added to the matrix constitution. This can be attributed to the increase in tungsten-matrix interfacial strength by the dissolution and of brittle intermetallic phases. It can be concluded that tungsten heavy alloys, particularly when cobalt is added as an alloying element should be used in the heat treated state. The effect of heat treatment is thus attributed to the dissolution of the brittle phases that may form on the $M / W$ interface.
\end{abstract}

Keywords: Tungsten Heavy Alloy, Heat Treatment, Intermetallics, Interfacial Strength.

\section{INTRODUCTION}

Tungsten based heavy alloys are two phase composites produced by LPS of mixed elemental tungsten, nickel, iron and/or cobalt powders. The sintered product is a composite consisting of tungsten and solidified matrix (W-Ni-Fe-Co) phases. These alloys are most useful in applications requiring high density, strength, and toughness.

The microstructure of LPS tungsten based alloys comprises a continuous network of approximately spheroidal tungsten single crystals (20 to $50 \mu \mathrm{m}$ in diameter) embedded in a ductile, nickel-based matrix phase. This composite structure results in high density materials with a useful combination of mechanical properties. Their properties are, however, observed to vary markedly with variations in composition, sintering conditions and post sintering treatments.

Several studies $[1,2,3,4]$ shown that, the mechanical properties of sintered WHA could be improved by heat treatment at about $1000^{\circ} \mathrm{C}$. This effect has been attributed either to the phase changes occurring in the alloy system [5] or eliminate hydrogen embrittlement in case of using hydrogen as a sintering atmosphere during sintering [4]. The heat treatments were mostly performed at $1000^{\circ} \mathrm{C}$ for fixed times (about $1 \mathrm{~h}$ ).

Heat treatment can be used to effectively break down the intermetallic formed in WHA by the interaction of tungsten and any of the binder constituents, particularly, when cobalt is added to strengthen the alloy matrix. Most of these intermetallic are not stable above $1000^{\circ} \mathrm{C}$. Quenching from a homogeneous solid solution temperature of about $1100^{\circ} \mathrm{C}$ can retain the ductile austenitic matrix in the alloy and hinder the formation of a second brittle phase.

The purpose of this investigation is to study effect of post sintering heat treatment on the mechanical properties and fracture behavior of WHA. 


\section{EXPERIMENTAL WORK}

The main constituents of the adopted tungsten heavy metal alloy are commercial pure tungsten, nickel, cobalt and iron powders. The characteristics of used are shown in previous researches [6].

For this objective, three different compositions were prepared as follows: The first alloy is the conventional adopted alloy, having the composition $93 \% \mathrm{~W}-4.9 \% \mathrm{Ni}-2.1 \%$ $\mathrm{Fe}$. The second alloy has the composition $91 \% \mathrm{~W}-6 \% \mathrm{Ni}-$ $3 \% \mathrm{Co}$, where, the iron is substituted completely with cobalt, which represents about $33 \%$ of the amount of the binder. The third alloy has the composition $90.5 \% \mathrm{~W}-5.6 \% \mathrm{Ni}-1.4 \%$ $\mathrm{Fe}-1.5 \% \mathrm{Co}$, where the cobalt represents about $15 \%$ of the amount of binder, while, the Ni:Fe ratio was 7:3. The W-Ni$\mathrm{Co}$ and the W-Ni-Fe-Co alloys are compared to the conventional $\mathrm{W}-\mathrm{Ni}-\mathrm{Fe}$, in a first stage, in the as-sintered condition.

All compositions of powders were homogeneously blended, shaped into standard tensile and impact test specimens and subjected to an uni-axial compaction pressure of $200 \mathrm{MPa}$. All the compacted samples were also sintered in a vacuum atmosphere at $1500^{\circ} \mathrm{C}$ for a period of 60 minutes in accordance with the sintering cycle applied by M.Sallam et al.[6], and finally allowed to cool in furnace to room temperature.

The sintered specimens are then heat treated, at $1100^{\circ} \mathrm{C}$ for one hour, in an atmosphere of flowing argon gas, using a tube furnace equipped with a special push rod which can allow locating the samples in the prescribed heating zone, as shown in Fig.(1). After heating, the samples are then immediately quenched in water.

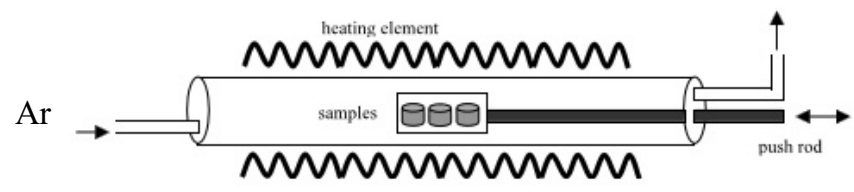

Fig -1: Schematic diagram of the tube furnace used in the applied heat treatment.

In this heat treatment, the soaking temperature should be sufficient to enable the intermetallic, which may be formed and persist at the $\mathrm{M} / \mathrm{W}$ interface during slow cooling from the sintering temperature, to diffuse into the austenitic phase, leaving this interface substantially or essentially free of intermetallic [5]. This desired material state is preserved by quenching the heat treated sample in oil, water or brine. Unless the samples are rapidly cooled in this manner, the desired mechanical properties are not obtained. Moreover, the application of this type of heat treatment has also an important beneficial effect, in the case when using hydrogen as a reducing atmosphere during sintering, since the flowing argon forces the residual hydrogen contained in the sintered specimen to diffuse out, consequently reducing the probability of occurrence of hydrogen embrittlment.
The samples with different compositions were tested in the as-sintered condition in an identical procedure explained by M.Sallam et al.[6], and the following properties were determined.

\section{RESULTS AND DISCUSSIONS}

\subsection{Mechanical Properties}

In order to evaluate the effect of heat treatment and the alloy chemical composition on the mechanical and structural properties of these compositions, the strength, elongation, impact resistance and hardness of samples having the different adopted compositions, were evaluated. The stress strain diagrams of the heat treated samples compared to those obtained in the as sintered state are shown in Fig-.(2), (3) and (4). The improvement in the tensile properties after heat treatment is evident for all chemical compositions.

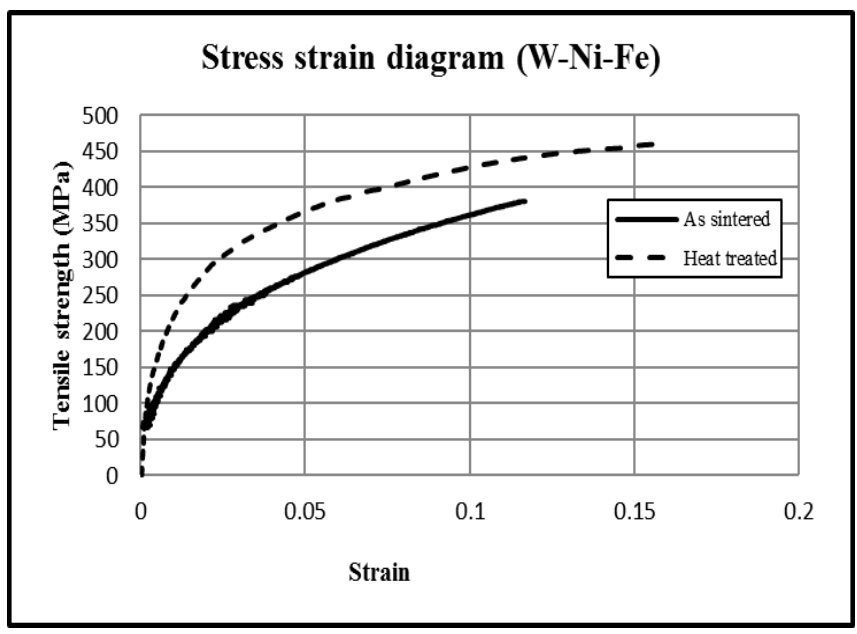

Fig -2: Stress strain diagram of the W-Ni-Fe heavy alloy in the heat treated state compared to that obtained in the as sintered state.

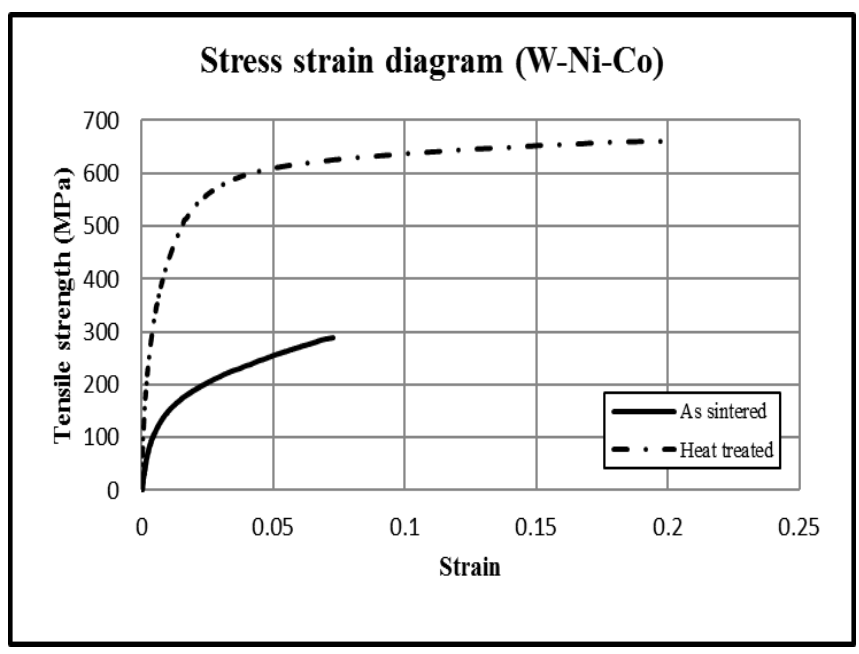

Fig -3: Stress strain diagram of W-Ni-Co heavy alloy in the heat treated state compared to that obtained in the as sintered state. 


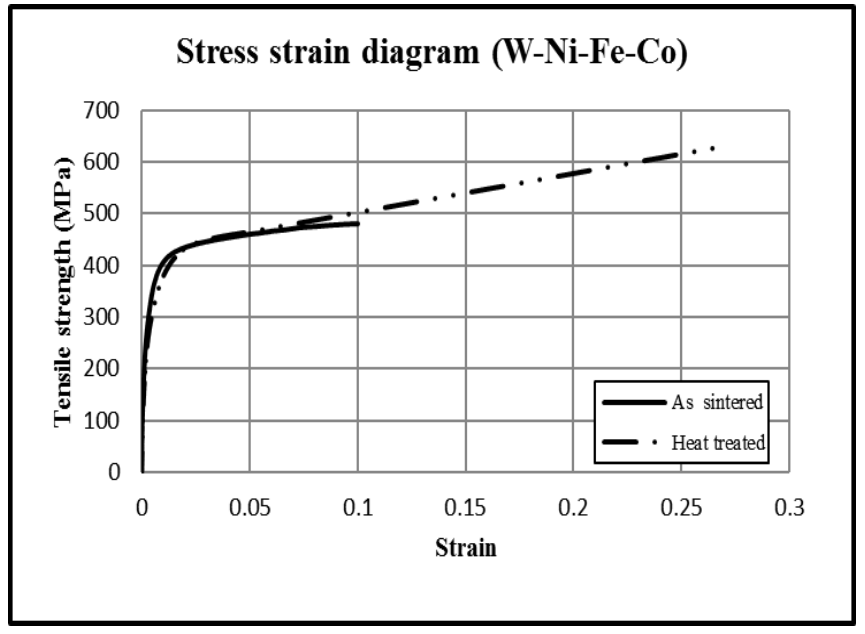

Fig -4: Stress strain diagram of W-Ni-Fe-Co heavy alloy in the heat treated state compared to that obtained in the as sintered state.

It is clearly visible that both strength and ductility of the heat treated samples, are increased for all the alloy compositions. In case of the W-Ni-Fe heavy alloy, the strength is increased by about $17.4 \%$, and the ductility by about $26.25 \%$ relative to its value in the as sintered state. While, in the case of the W-Ni-Fe-Co heavy alloy, the strength of the heat treated samples increased by about $23.8 \%$, and the ductility by about $63 \%$. In this case, the ductility is increased in a very pronounced manner, while the strength gained an increase of about quarter the strength at the sintered state. In the third alloy (W-Ni-Co heavy alloy), where cobalt completely replaces iron in the alloy composition, an attractive increase in both strength and ductility attained $56 \% \quad 63.5 \%$ respectively. This clearly indicates that this tungsten heavy alloys, particularly when cobalt is added as a matrix ingradient, should be used in the heat treated conditions to make use of the beneficial effect of these heat treatments in dissolution of the brittle phases that may form between the $\mathrm{M} / \mathrm{W}$.

The mutual interactive effects of the application of post heat treatment and the addition of cobalt to the alloy composition on the tensile properties can be summarized in the bar charts illustrated in Figs.(5) and (6). We can note that the addition of cobalt improves the tensile strength in a first stage then it drastically decreases this strength in a second stage, on the other hand, it monotonically decreases ductility with increasing its content in the as sintered state. Furthermore, we can remark that after the application of the prescribed heat treatment, the strength increases continuously with increasing the cobalt content to attain a value of $660 \mathrm{MPa}$ when the cobalt completely replaces iron in the matrix.

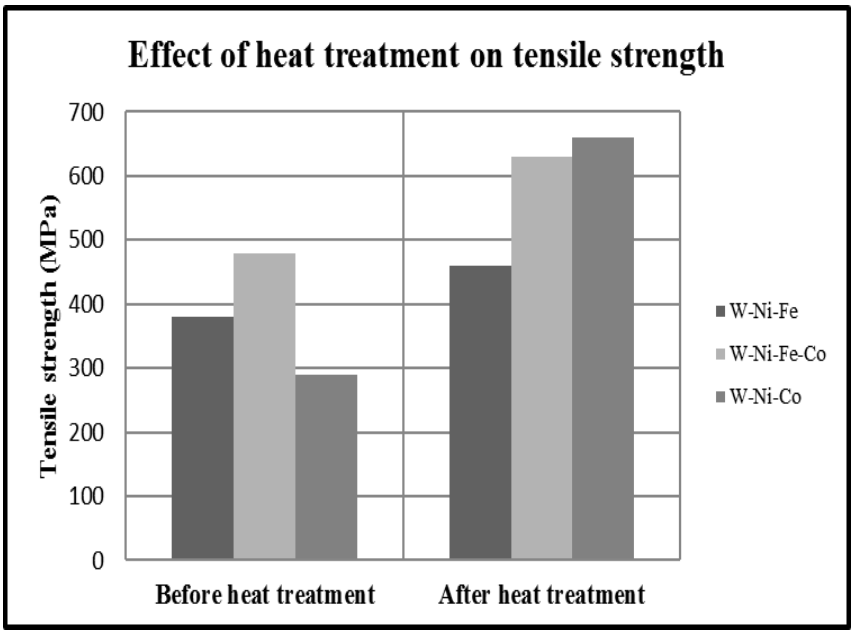

Fig -5: Effect of heat treatment and chemical composition on tensile strength of tungsten heavy alloys uni-axially compacted under $200 \mathrm{MPa}$ and sintered in vacuum at $1500^{\circ} \mathrm{C}$ for 60 minutes.

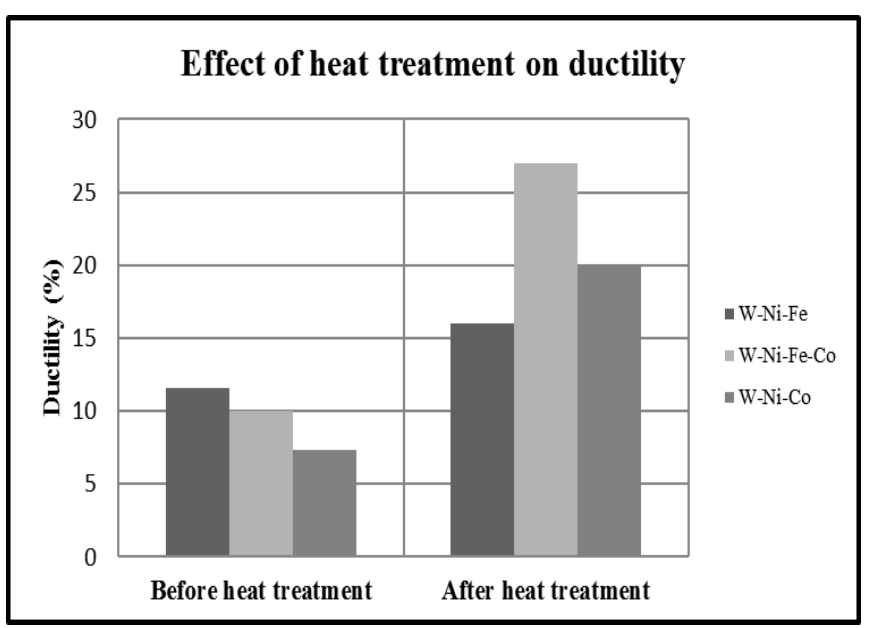

Fig -6: Effect of heat treatment and chemical composition on ductility of tungsten heavy alloys uni-axially compacted under $200 \mathrm{MPa}$ and sintered in vacuum at $1500^{\circ} \mathrm{C}$ for 60 minutes.

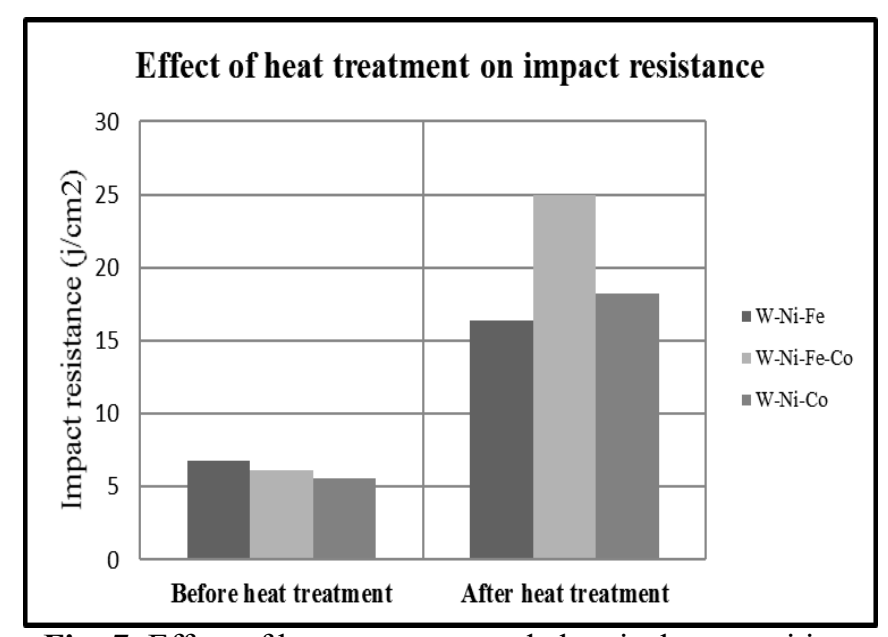

Fig -7: Effect of heat treatment and chemical composition on impact resistance of tungsten heavy alloys uni-axially compacted under $200 \mathrm{MPa}$ and sintered in vacuum at $1500^{\circ} \mathrm{C}$ for 60 minutes. 
Regarding the effect of heat treatment on the hardness, we can note a slight increase for all adopted alloys as shown in Fig.(8). The hardness of the heat treated samples, is increased only by about $5.5 \%$ in case of the W-Ni-Fe alloy, and by about $7.7 \%$ in case of the W-Ni-Fe-Co and W-Ni-Co alloys. This slight increase in hardness can be attributed to the fact that, hardness is a surface property, which is much less affected by the presence of intermetallic brittle phases on the boundaries of tungsten grains, and the nature of the interfacial bond between tungsten particles and matrix.

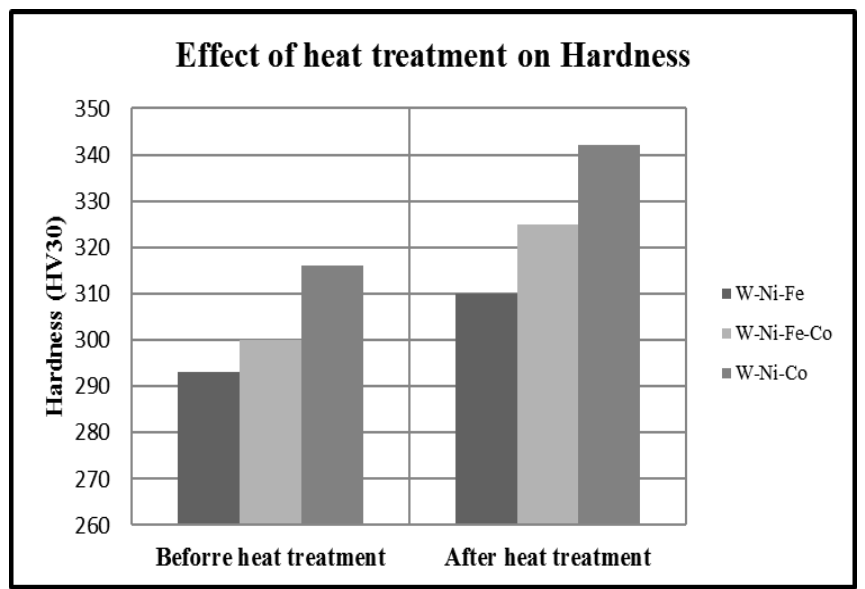

Fig -8: Effect of heat treatment and chemical composition on hardness of tungsten heavy alloys uni-axially compacted under $200 \mathrm{MPa}$ and sintered in vacuum at $1500^{\circ} \mathrm{C}$ for 60 minutes.

Here, we can conclude that the heat treated alloys can provide improved mechanical properties and can be considered suitable candidate for applications that depend on high stress, like improving the ballistic performance for the kinetic energy penetrators, where the ductility enough satisfactory for these applications [7].

\subsection{Fracture Behavior}

The investigated micrographs of fracture surfaces, taken by scanning electron microscope at two different locations, of the heat treated samples having the indicated different chemical compositions are shown in Figs.(9), (10) and (11).

All the modes of fracture can be clearly observed in these micrographs, however, the close examination of the fracture surfaces taken on the specimens of W-Ni-Fe based heavy alloys reveals a dominant failure by interfacial separation along the tungsten-matrix interface or by matrix rupture as shown in Fig.(9). Moreover, when cobalt is added to the alloy together with the other binding ingredients (nickeliron) an important proportions of tungsten particles cleavage appears together with the other separation modes as shown in Fig.(10). This observation is in accordance with the recorded increase of tensile strength of such alloy, in the heat treated state, relative to the $\mathrm{W}-\mathrm{Ni}-\mathrm{Fe}$ alloy only as shown in Fig.(5). Furthermore, when cobalt completely replaces iron in the alloy composition, cleavage becomes the dominant failure mode in the obtained fracture micrographs as shown in Fig.(11). This indicates that the tungsten-matrix interfacial strength is significantly improved specially after the application of heat treatment that allows the dissolution and prevention of the formation of brittle intermetallic phases.
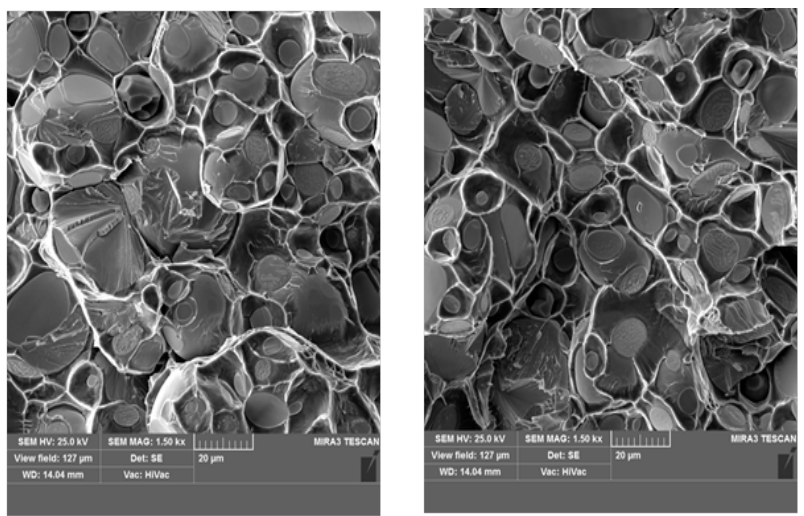

Fig -9: Tensile fracture surfaces taken at two different locations of $93 \% \mathrm{~W}-4.9 \% \mathrm{Ni}-2.1 \% \mathrm{Fe}$ heavy alloy uni-axially compacted under $200 \mathrm{MPa}$, sintered at $1500^{\circ} \mathrm{C}$ for 60 minutes, and heat treated at $1100^{\circ} \mathrm{C}$ for 1 hour then water quenched.
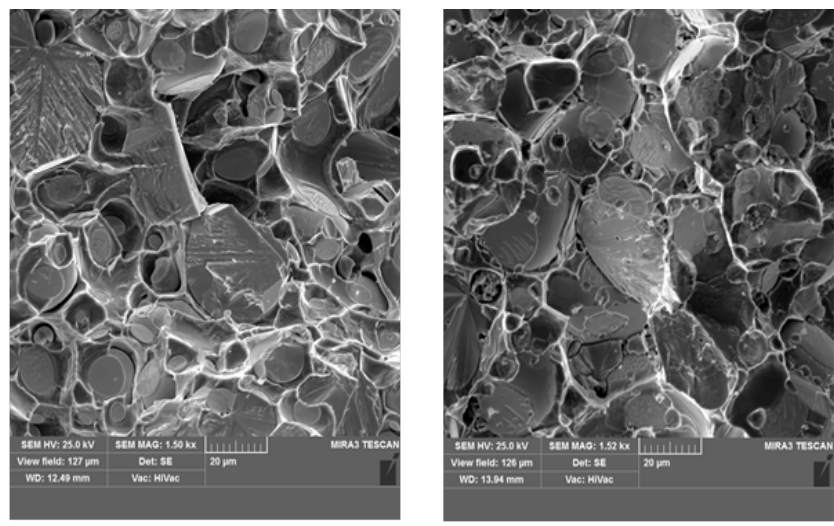

Fig -10: Tensile fracture surfaces taken at two different locations of $90.5 \% \mathrm{~W}-5.6 \% \mathrm{Ni}-2.4 \% \mathrm{Fe}-1.5 \% \mathrm{Co}$ heavy alloy uni-axially compacted under $200 \mathrm{MPa}$, sintered at $1500^{\circ} \mathrm{C}$ for 60 minutes, and heat treated at $1100^{\circ} \mathrm{C}$ for 1 hour then water quenched.
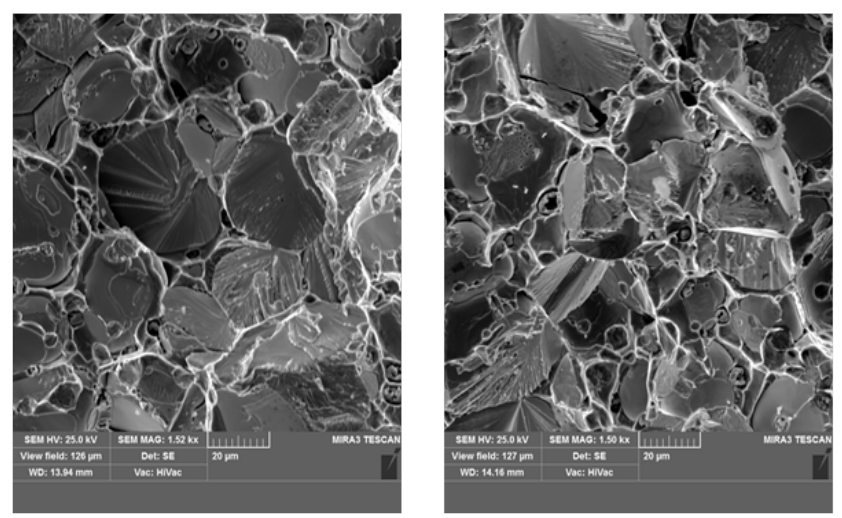

Fig -11: Tensile fracture surfaces taken at two different locations of $91 \% \mathrm{~W}-6 \% \mathrm{Ni}-3 \% \mathrm{Co}$ heavy alloy uni-axially compacted under $200 \mathrm{MPa}$, sintered at $1500^{\circ} \mathrm{C}$ for 60 minutes and heat treated by heating to $1100^{\circ} \mathrm{C}$, holding for 1 hour then water quenched. 
The increase of elongation and UTS by the application of a post heat treatment cycle measured on the $\mathrm{W}-\mathrm{Ni}-\mathrm{Fe}$ is in accordance with the results of similar composition investigated by D.N.Yoon et al. [3]. They studied the effect of heat-treatment in vacuum and hydrogen on the ductility and UTS of the $96 \mathrm{~W} \%-2.8 \mathrm{Ni} \%-1.2 \mathrm{Fe} \%$. The results show that, elongation and the UTS increased significantly over the temperature range between $400-800^{\circ} \mathrm{C}$. Furthermore, at $1000^{\circ} \mathrm{C}$, there is a pronounced increase in elongation and UTS after vacuum heat treatment. On the other hand, hydrogen heat treatment has no effect on elongation due to effect of hydrogen embrittlement.

It was also reported by Sunghak Lee et al. [8] that in order to prevent hydrogen embrittlement resulting from sintering under hydrogen atmosphere and interfacial segregation of impurities, a post sintering heat treatment cycle is applied for tungsten heavy alloy. They applied a heat treatment cycle under vacuum for 5hours at $1150^{\circ} \mathrm{C}$ (temperature slightly higher than the temperature of formation of intermetallic compounds), for the heavy alloy $93 \mathrm{~W} \%-$ $4.9 \mathrm{Ni} \%-2.1 \mathrm{Fe} \%$ after being LPS with hydrogen atmosphere, and then water cooled. They also applied repetitive cycles at $1180^{\circ} \mathrm{C}$ for 2 hours, followed by water cooling, and at $1200^{\circ} \mathrm{C}$ for 1 hour followed water quenching. It was found that toughness is significantly improved after this three steps heat treatment.

In a similar research, Penrice [5] indicated that the ductility of W-Ni-Fe alloys can be improved by heat treatment by promoting the dissolution of any brittle intermetallic phases that may be present at interfaces. W.D.Cai et al. [4] stated that post sintering heat treatment of tungsten heavy alloys is often performed in an effort to homogeneously redistribute any impurities present and to prevent the formation of intermetallic phases at interfaces, besides eliminating residual hydrogen (or degassing) in case of sintering under hydrogen atmosphere, thereby, preventing hydrogen embrittlement.

\section{CONCLUSION}

The main contribution of this study is the definitive experimental demonstration that the mechanical properties of WHA are improved after applying a post sintering heat treatment cycle in argon, especially if cobalt is added as an alloying element. In the present study, mechanical tests were conducted on WHA specimens fabricated by sintering and heat treatment. Conclusions are summarized as below:

- Under quasi-static loading, the heat treated samples show a considerable amount of increase in strength and ductility, for all the alloy compositions.

- A remarkable improvement in impact resistance for all adopted chemical compositions is noticed for samples in the heat treated condition.

- A slight increase in hardness for all adopted alloys is measured for the heat treated samples. This slight increase in hardness can be attributed to the fact that, hardness is a surface property, which is much less affected by the presence of intermetallic brittle phases on the boundaries of tungsten grains.
- The addition of cobalt improves the tensile strength, in a first stage, then it decreases this strength in a second stage, while, the ductility decreases continuously with increasing cobalt content in the as sintered state. Furthermore, After the application of the prescribed heat treatment, the strength increases continuously with increasing the cobalt content.

- When cobalt is added as a matrix ingradient for tungsten heavy alloys, the alloy should be used in the heat treated state, to allow the dissolution of the brittle phases that may form on the interface between $\mathrm{M} / \mathrm{W}$ interface.

- Observation of fracture surfaces shows that, cobalt content plays an important role in dictating the fracture mode during tensile testing. In $\mathrm{W}-\mathrm{Ni}-\mathrm{Fe}$ interfacial separation along the tungsten-matrix interface or matrix rupture are the dominant failure modes. When cobalt is added, together with iron to the matrix ingradients, tungsten cleavage together with matrix failure are dominant modes. As the cobalt content increases, and completely replaces iron in the alloy composition, cleavage becomes the dominant failure mode.

- It can be concluded that the tungsten-matrix interfacial strength is significantly improved specially after the application of heat treatment that allows the dissolution and prevention of the formation of brittle intermetallic phases.

\section{REFERENCES}

[1]. J.M.Sakai and C.Grabarek, "Powder Metallurgy in Defense Technology", Vol.4, pp.85, (MPIF, Princeton), 1978.

[2]. D.V.Edmonds and P. N.Jones, Metallurgical Tranactions, Vol.10A, pp.289, 1979.

[3]. H.K.Yoon, S.H.Lee, L.Kang, D.N.Yoon, "Effect of vacuum-treatment on mechanical properties of $\mathrm{W}-\mathrm{Ni}-\mathrm{Fe}$ heavy alloy", Journal of materials science, Vol.18, pp. 1374-1380, 1983.

[4]. W.D.Cai, Yi Li, R.J. Dowding and F.A. Mohamed, "A review of tungsten based alloys as kinetic energy penetrator materials", Third international conference on tungsten and refractory metals, edited by A.bose and J.Dowding, 1995. [5]. Penrice et al., "High density tungsten-nickel-iron-cobalt alloys having improved hardness and methods for making same", U.S patent, Patent number 4,762,559, Aug.1988.

[6]. A.S.Abdallah, A. Fayed, G.Abdou, M.Tolba Sallam, "Effect of processing parameters on the mechanical and structure properties of $93 \mathrm{~W}-4.9 \mathrm{Ni}-2.1 \mathrm{Fe}$ Tungsten heavy alloy", Fifteenth International Conference on Aerospace Sciences \& Aviation Technology, ASAT-15, MTC, Cairo, 2013.

[7]. Anish Upadhyaya, "Processing strategy for consolidating tungsten heavy alloys for ordnance applications", Materials Chemistry and Physics, Vol.67, pp. 101-110, 2001.

[8]. Don-Kuk Kim, Sunghak Lee, Joon-Woong Noh, "Dynamic and quasi-static torsional behavior of tungsten heavy alloy specimens fabricated through sintering, heattreatment, swaging and aging", Materials Science and Engineering, Vol. A247, pp.285-294, 1998. 